\title{
All-optical modulation in a CMOS-compatible amorphous silicon-based device
}

\author{
S. Rao \\ sandro.rao@unirc.it \\ C. D'Addio
}

F. G. Della Corte

\begin{abstract}
Department of Information Science, Mathematics, Electronics and Transportations (DIMET) Università degli Studi "Mediterranea", Via Graziella Località Feo di Vito, Reggio Calabria, 89060, Italy

Department of Information Science, Mathematics, Electronics and Transportations (DIMET) Università degli Studi "Mediterranea", Via Graziella Località Feo di Vito, Reggio Calabria, 89060, Italy Institute for Microelectronics and Microsystems - Consiglio Nazionale delle Ricerche (IMM-CNR) Unit of Napoli, Via P. Castellino, 111, 80132, Napoli, Italy

Department of Information Science, Mathematics, Electronics and Transportations (DIMET) Università degli Studi “Mediterranea”, Via Graziella Località Feo di Vito, Reggio Calabria, 89060, Italy

Institute for Microelectronics and Microsystems - Consiglio Nazionale delle Ricerche (IMM-CNR) Unit of Napoli, Via P. Castellino, 111, 80132, Napoli, Italy
\end{abstract}

Active silicon photonic devices, which dynamically control the flow of light, have received significant attention for their use in on-chip optical networks. High-speed active silicon photonic modulators and switches rely on the plasma dispersion effect, where a change in carrier concentration causes a variation in the refractive index. The necessary electron and hole concentration change can be introduced either by optical pumping, or by direct electrical injection and depletion. We demonstrate a fast photoinduced absorption effect in low loss hydrogenated amorphous silicon (a-Si:H) waveguides deposited at a temperature as low as $190^{\circ} \mathrm{C}$. Significant modulation ( $\mathrm{M} \% \sim 90 \%$ ) occurs with a $1 \mathrm{~mm}$-long device. We attribute the enhanced modulation to the significantly larger free-carrier absorption effect of a-Si:H. The complementary metal-oxide semiconductor (CMOS) compatible technology of a-Si:H could be considered as a promising candidate to enable an easy back-end integration with standard microelectronics processes.

[DOI: http://dx.doi.org/10.2971/jeos.2012.12023]

Keywords: amorphous silicon, integrated optics, silicon optoelectronics, free carrier absorption, waveguides

\section{INTRODUCTION}

Photonics is a rapidly growing sector in the global economy. Optical communications, optical storage, imaging, lighting, optical sensors or security are just a few examples. Even if photonics could bring new functionalities to electronic components, e.g. low propagation losses, high bandwidth, wavelength multiplexing and immunity to electromagnetic noise, the high cost of photonic components is a major obstacle to their deployment in most of the application fields. In microelectronics, many applications can be realised in a much more compact and cost-effective way by integrating the required functionality in a single chip. Photonics on CMOS seems to be the way to tackle such issue by developing a small number of generic integration technologies with a level of functionality that can address a broad range of applications [1].

Hydrogenated amorphous silicon (a-Si:H), deposited using the CMOS-compatible low temperature $\left(120-400^{\circ} \mathrm{C}\right)$ Plasma Enhanced Chemical Vapour Deposition (PECVD) technique, is recently emerging as a useful material for realising minimally-invasive on-chip passive [2]-[4] and active [5]-[8] devices for optical interconnects. In this paper we report experimental results on all-optical modulation in a compact device. Modulation is achieved by using short optical pump laser pulses to induce an absorption change in the a-Si:H waveguide.

\section{WAVEGUIDE STRUCTURE AND FABRICATION}

A schematic cross section of the designed waveguide is shown in Figure 1. The device, realised on a p-doped crystalline silicon (c-Si) substrate $(\rho=0.001 \Omega \cdot \mathrm{cm})$, consists of a rib waveguide made of a 3- $\mu$ m-thick a-Si:H undoped layer (refractive index $n_{a-S i: H}=3.58$ ) on a $400 \mathrm{~nm}$-thick $\mathrm{SiO}_{2}$ low refractive index cladding $\left(n_{\mathrm{SiO} 2}=1.45\right)$ ensuring a vertical optical confinement. The undoped a-Si:H layer is deposited from the plasmaassisted decomposition of $\mathrm{SiH}_{4}$ at a flow of $20 \mathrm{sccm}$ (standard cubic centimetres per minute) and an RF power of $\mathrm{P}_{R F}=4 \mathrm{~W}$ at the frequency of $f=13.56 \mathrm{MHz}$. The maximum temperature during the process was as low as $190^{\circ} \mathrm{C}$, which makes it fully compatible with standard CMOS processes. The CMOSfriendly technological deposition process allows, in principle, the simple fabrication of a photonic layer on top of the electronic microchip at the end of the CMOS flow, just before encapsulation. 


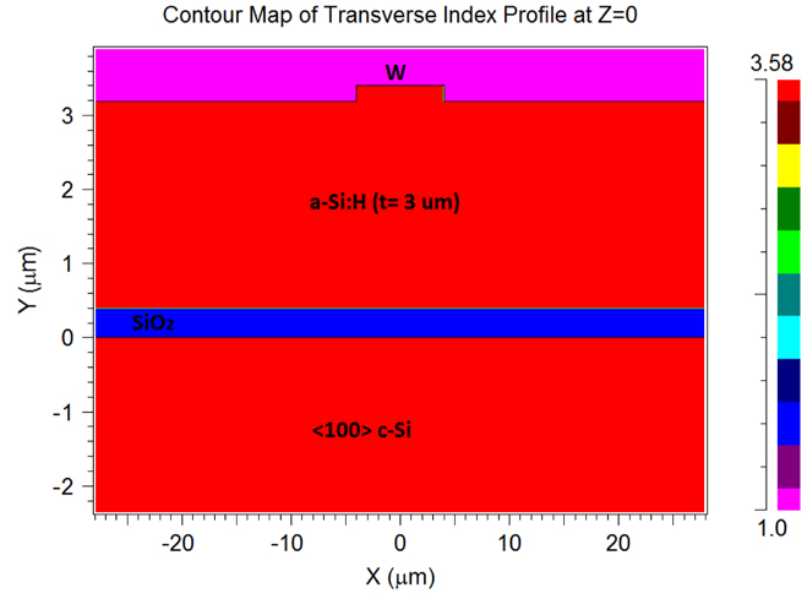

FIG. 1 Schematic cross section of the realised waveguides and corresponding refractive index colour bar. The crystalline silicon substrate is $300 \mu \mathrm{m}$ thick.

Standard technological processes have been used, i.e. optical lithography and reactive ion etching (RIE), to realise the rib waveguide. Optical simulations [9] show that the designed waveguide supports only one mode for TE and TM polarisation once a $8 \mu \mathrm{m}$-wide and $210 \mathrm{~nm}$-high rib is defined while the first-order higher modes, $\mathrm{TE}_{01}$ and $\mathrm{TM}_{01}$, are localised in the slabs, far from the rib.

\section{ALL-OPTICAL EXPERIMENTAL RESULTS}

We have tested the behavior of the a-Si:H-based waveguiding active device by inducing a strong photoinduced absorption on a $1.0 \mathrm{~mm}$-long device using a pump-probe optical setup. In such configuration, while the pump beam is orthogonal to the film, the probe beam is launched transversally to the film thickness and guided by the waveguide core, increasing in this way the interaction length between the active material and the optical signal.

In our experiments, the waveguide propagation losses were first measured by the cut-back technique. Several samples with lengths in the range from 800 to $1500 \mu \mathrm{m}$ were cut from the substrate by cleavage. Sample facets did not receive polishing treatment. From these samples we calculated a loss coefficient of $1.3 \pm 0.1 \mathrm{~dB} / \mathrm{cm}\left(0.29 \pm 0.02 \mathrm{~cm}^{-1}\right)$. The overall insertion losses of the devices are $\sim 10 \mathrm{~dB}$ with the main contribution due to the coupling losses, a drawback of the high refractive index of a-Si:H used as core material.

The experimental setup is depicted in Figure 2. A $4 \mathrm{~mW}$ laserdiode probe radiation at the wavelength of $1550 \mathrm{~nm}$ was coupled into the device via a lensed fiber. The transmitted light was collected at the chip output by a multi-mode fiber and detected by a $2 \mathrm{GHz}$ bandwidth InGaAs photodiode. We used the $2^{\text {nd }}$ harmonic $\left(\lambda_{\text {PUMP }}=532 \mathrm{~nm}\right)$ of a high energy Nd:YAG laser for inducing the free carrier injection in the waveguide. The low level optical probe signal at the waveguide output was recovered by mean of an erbium doped fiber amplifier (EDFA).

Optical modulation is achieved by using short optical pump

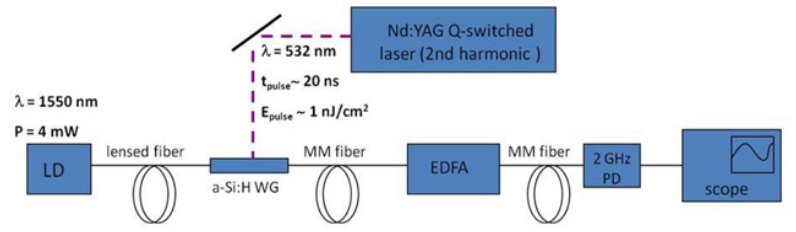

FIG. 2 Experimental setup used for the characterisation of the photoinduced absorption effect in an a-Si:H layer.

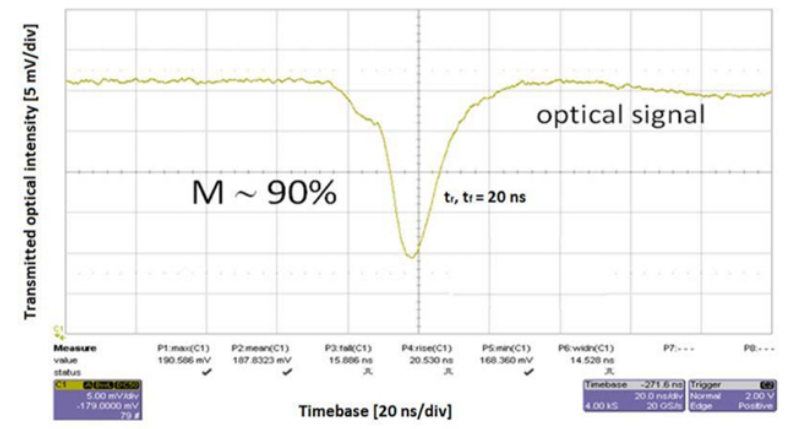

FIG. 3 Photoinduced absorption effect induced in a $3 \mu \mathrm{m}$-thick, 1-mm-long, a-Si:H rib waveguide. The electrical signal at the photodiode output is shown in the figure. The pump pulse was a high-energy, $532 \mathrm{~nm}$ wavelength, 20-ns-long, coherent light pulses. The measured rise and fall times are $\sim 20$ ns and the modulation depth is $90 \%$.

pulses $\left(\mathrm{E}_{\text {pulse }} \sim 1 \mathrm{~nJ} / \mathrm{cm}^{2}\right.$ ) to induce a change in the extinction coefficient, $k$, of the amorphous waveguiding material. We attributed the continuous-wave laser modulation to the free carriers absorption effect induced by the generated photo-excited carriers (the quasi-bandgap of a-Si:H is $\sim 1.75 \mathrm{eV}$ ). We measured a modulation depth, defined as: $\left(\mathrm{I}_{M A X}-\mathrm{I}_{M I N}\right) / \mathrm{I}_{M A X}$ where $I_{M A X}$ and $I_{M I N}$ are the maximum and minimum intensities of the transmitted signal, of $\mathrm{M}_{\%} \sim 90 \%$ when an incident pump pulse is delivered. Due to the poor overlap of the pump light with the a-Si:H active region, which is mainly confined under the rib waveguide (Active Area $\sim 8 \mu \mathrm{m} \times 1 \mathrm{~mm}$ ) where the probe beam propagates, we estimated that only $80 \mathrm{fJ}$ of the pump energy contributes to the absorption effect. Consequently, we are confident that the modulation depth could be improved with a better overlap even providing a lower pump pulse energy. It is worth noting that no Fabry-Perot interference effect from the ends facets were observed in transmission measurements.

As shown in Figure 3, the probe optical beam at the photodiode output falls when the pump photons are absorbed. In fact, since the photon energy is much larger than the quasibandgap energy of a-Si: $\mathrm{H}$, the electrons are excited into the conduction band highly increasing the free carrier absorption effect. We measured rise and fall time of the order of $20 \mathrm{~ns}$, which are higher than what observed in $\mathrm{c}-\mathrm{Si}$, where characteristic times of the order of hundreds of picoseconds have been observed [10]. We note, however, that in our experiments the characteristic times are probably limited by the dynamics of our Nd:YAG pump beam. From the degree of modulation measured in our experiment, we estimated an absorption coefficient variation $\Delta \alpha \sim 23 \mathrm{~cm}^{-1}$.

The refractive index change in silicon, due to injection or depletion of free carrier at $\lambda=1.55 \mu \mathrm{m}$, can be derived to a first 
order approximation from the classical Drude model also for amorphous silicon semiconductor [11], in particular the absorption coefficient variation is given by:

$$
\Delta \alpha=\frac{e^{3} \lambda^{2}}{4 \pi^{2} c^{3} \varepsilon_{0} n}\left(\frac{\Delta N_{e}}{m_{e}^{2} \mu_{e}}+\frac{\Delta N_{h}}{m_{h}^{2} \mu_{h}}\right)
$$

In Eq. (1), $e$ is the electron charge, $\lambda$ is the probe wavelength, $\varepsilon_{0}$ is permittivity of free space, $n_{0}$ is the refractive index of the material, $m_{e}$ and $m_{h}$ are the effective masses of electrons and holes, $\mu_{e}$ and $\mu_{h}$ are the mobilities of the carriers in aSi:H. Substituting for $m_{e}=0.5 \cdot m_{0}, m_{h}=1.0 \cdot m_{0}, m_{0}=9.1 \cdot 10^{-31} \mathrm{~kg}$, $\mu_{e}=2.0 \mathrm{~cm}^{2} / \mathrm{V} \cdot \mathrm{s}, \mu_{h}=0.4 \mathrm{~cm}^{2} / \mathrm{V} \cdot \mathrm{s}$ [11] in Eq. (1), yields a theoretically estimated average free carriers concentration change of:

$$
\Delta N_{e} \sim \Delta N_{h}=1.4 \times 10^{17} \mathrm{~cm}^{-3} .
$$

\section{CONCLUSION}

We have demonstrated an all-optical absorption-induced modulation of light in an amorphous silicon waveguide based on the free carrier effect using a pump-probe technique. The optical signal turn-on/turn off transients were measured to be of $\sim 20 \mathrm{~ns}$, limited however by the pump laser source dynamic characteristics. The modulation depth is $90 \%$ when an incident pump pulse with energy of $E_{\text {pump }} \sim 80 \mathrm{fJ}$ is absorbed in the active waveguiding region.

Technologically, the fabrication process involves temperatures below $190^{\circ} \mathrm{C}$, which are compatible with standard microelectronic processes and in particular suitable for the realisation of a photonic layer on top of an integrated circuit.

\section{ACKN OWLEDGEMENTS}

The research leading to these results has received funding from the European Community's Seventh Framework Programme (FP7/2007-2013) under grant agreement n ${ }^{\circ} 224312$ HELIOS.

\section{References}

[1] J. M. Fedeli, L. Di Cioccio, D. Marris-Morini, L. Vivien, R. Orobtchouk, P. Rojo-Romeo, C. Seassal, and F. Mandorlo, "Development of silicon photonics devices using microelectronic tools for the integration on top of a CMOS wafer," Advances in Optical Technologies 2008, 1-15 (2008).

[2] G. Cocorullo, F.G. Della Corte, R. De Rosa, I. Rendina, A. Rubino, and E. Terzini, "Amorphous silicon-based guided-wave passive and active devices for silicon integrated optoelectronics," IEEE J. Sel. Top. Quantum Electron. 4, 997-1001 (1998).

[3] A. Harke, M. Krause, and J. Mueller, "Low-loss single mode amorphous silicon waveguides," Electronics Lett. 41, 1377-1378 (2005).

[4] S. K. Selvaraja, E. Sleeckx, M. Schaekers, W. Bogaerts, D. Van Thourhout, P. Dumon, and R. Baets, "Low-loss amorphous siliconon-insulator technology for photonic integrated circuitry," opt. Comm. 282, 1767-1770 (2009).

[5] K. Narayanan, A. W. Elshaari, and S. F. Preble, "Broadband alloptical modulation in hydrogenated-amorphous silicon waveguides," Opt. Express 18, 10 (2010).

[6] F. G. Della Corte, S. Rao, M. A. Nigro, F. Suriano, and C. Summonte, "Electro-optically induced absorption in a-Si:H/a-SiCN waveguiding multistacks," Opt. Express 16, 7540-7550 (2008).

[7] S. Rao, F. G. Della Corte, C. Summonte, and F. Suriano, "Electrooptical modulating device based on a CMOS-compatible a-Si:H/aSiCN multistack waveguide," IEEE J. Sel. Top. Quantum Electron. 16(1), 173-178 (2010).

[8] F. G. Della Corte, S. Rao, G. Coppola, and C. Summonte, "Electrooptical modulation at $1550 \mathrm{~nm}$ in an as-deposited hydrogenated amorphous silicon p-i-n waveguiding device," Opt. Express 19(4), 2941-2951 (2011).

[9] RSoft Photonics CAD Suite version 5.1.7, by RSOFT Inc.

[10] V. R. Almeida, C. A. Barrios, R. R. Panepucci, and M. Lipson, "All-optical control of light on a silicon chip," Nature 431(7012), 1081-1084 (2004).

[11] P. M. Fauchet, D. Hulin, R. Vanderhaghen, A. Mourchild, and W. L. Nighan Jr., "The properties of free carriers in amorphous silicon," J. Non-Cryst. Sol. 141, 76-87 (1992). 